\title{
ON AN ANALOG OF LAGRANGE'S THEOREM FOR COMMUTATIVE HOPF ALGEBRAS
}

\author{
DAVID E. RADFORD ${ }^{1}$
}

\begin{abstract}
We show that the Hopf algebra $A$ over any field $k$ representing the affine group scheme SL(2, ) is not a free $B$-module for some sub-Hopf algebra $B$ of $A$. In particular $k$ can be algebraically closed, or of characteristic 0 in which case $A$ is also cosemisimple.
\end{abstract}

Introduction. Let $G$ be a finite group and suppose that $H$ is a subgroup of $G$. If $k$ is a field, then Lagrange's theorem can be formulated in terms of the group algebra $k[G]$, namely $k[G]$ is a free (left) $k[H]$-module. An obvious Hopf algebra analog would be the following: If $A$ is a Hopf algebra over a field $k$ and $B$ is a sub-Hopf algebra of $A$, then $A$ is a free (left) $B$-module. This is not generally true; there exist commutative cocommutative counterexamples over certain nonalgebraically closed fields [1]. By the theorem of [3], the field of definition of a cocommutative counterexample can never be algebraically closed.

In this paper we show that the Hopf algebra $A$ over a field $k$ representing the affine group scheme $\mathrm{SL}(2$,$) is a finitely generated B$-module which is not free for some sub-Hopf algebra $B$ of $A$. In particular, $k$ can be algebraically closed. In characteristic 0 observe that $A$ is cosemisimple [7]. Our proof is very elementary and reduces to simple computations in the polynomial ring $k[X]$.

It should be noted that a commutative Hopf algebra $A$ over a field $k$ is always a faithfully flat and projective $B$-module for any sub-Hopf algebra $B$ [5, Theorem 3.1] and [6, Corollary 1], and if $B$ is finite-dimensional then $A$ is a free $B$-module [3, Theorem 1]. For further results on the analog of Lagrange's theorem the reader is referred to [2], [3], [5], [6].

The main result. Let $k$ be a field and $n \geqslant 1$ a fixed integer. Let $C$ be the vector space with basis of symbols $X_{i j}, 1 \leqslant i, j \leqslant n$. Then $C$ is a coalgebra where $\Delta X_{i j}=\Sigma_{k} X_{i k} \otimes X_{k j}$ and $\varepsilon\left(X_{i j}\right)=\delta_{i j}$ for all $i, j$. The coalgebra structure on $C$ extends uniquely to a bialgebra structure on the symmetric algebra $S(C)$ of $C$. It is well known that the determinant form $d=\Sigma_{\sigma} \operatorname{sgn}(\sigma) X_{1 \sigma(1)} \cdot \ldots \cdot X_{n \sigma(n)}$ satisfies $\Delta d=d \otimes d$, and the quotient $A=S(C) /(d-1)$ has a Hopf algebra structure. It is easily seen that $A$ represents $\operatorname{SL}(n$,$) . For the remainder of this paper we let$ $n=2$.

Received by the editors June 9, 1979.

AMS (MOS) subject classifications (1970). Primary 16A24.

Key words and phrases. Hopf algebra, free module.

${ }^{1}$ The research for this paper was partially supported by NSF grant No. MCS77-00113 A01.

(C) 1980 American Mathematical Society 0002-9939/80/0000-0251/\$01.75 
Theorem. Let $k$ be any field and $A=(C)$ be as above. If $B=\left(C^{2}\right)$ is the subalgebra generated by $C^{2}$, then $B$ is a sub-Hopf algebra, and $A$ is a finitely generated $B$-module which is not free.

Proof. $C^{n}$ is a subcoalgebra for $n \geqslant 1$, so $B=k+C^{2}+C^{4}+\ldots$ is a sub-bialgebra. If $s$ is the antipode of $A$ then $s(C) \subset C$, so $B$ is in fact a sub-Hopf algebra. It is clear that $k+C$ generates $A=k+C+C^{2}+C^{3}+\ldots$ as a $B$-module. It remains to show that $A$ is not free. Let $M=C+C^{3}+C^{5}+\ldots$ Then $M$ is a $B$-module and:

(1) $A=B \oplus M$.

To prove (1) regard $S(C)=k\left[X_{11}, X_{12}, X_{21}, X_{22}\right]$ as a polynomial ring. Let $S(C)_{0}$ be the span of monomials of even degree and $S(C)_{1}$ be the span of monomials of odd degree $\left(X_{i j}\right.$ has degree 1). Then $S(C)=S(C)_{0} \oplus S(C)_{1}$, and if $S(C) \stackrel{\pi}{\rightarrow} A$ is the projection, we have $\pi\left(S(C)_{0}\right)=B$ and $\pi\left(S(C)_{1}\right)=M$. If $I \subset S(C)$ is the ideal generated by $d-1=X_{11} X_{22}-X_{12} X_{21}-1$, then $I=I_{0} \oplus I_{1}$ where $I_{i}=I \cap$ $S(C)_{i}$ for $i=0,1$. This proves (1).

(2) If $A$ is a free $B$-module then $M$ is a free rank $1 B$-module.

To prove this let $J=\operatorname{ker} \varepsilon_{B}$ be the augmentation ideal of $B$. Assume $A$ is a free $B$-module. Then $A / J A$ is a free $B / J$-module of the same rank. By (1) $A / J A \simeq$ $B / J \oplus M / J M$, so the rank of $A / J A$ as a $B / J \simeq k$-module is $1+\operatorname{dim} M / J M$.

We first show that $A$ has $\operatorname{rank} 2$ by showing $\operatorname{dim} M / J M=1$. Since $\varepsilon\left(x_{11}\right)=$ $\varepsilon\left(x_{22}\right)=1$ and $\varepsilon\left(x_{12}\right)=\varepsilon\left(x_{21}\right)=0$ (small case letters denote cosets of $A=$ $S(C) / I)$, and $M$ is spanned by cosets represented by monomials of odd degree, $M / J M$ is spanned by $\bar{x}_{11}, \bar{x}_{12}, \bar{x}_{21}$, and $\bar{x}_{22}$. Now multiplying the equation $x_{11} x_{22}-$ $x_{12} x_{21}=1$ by $x_{11}, x_{12}$, and $x_{21}$ respectively, we deduce that $x_{11}-x_{22}, x_{12}, x_{21} \in J M$. Therefore $\operatorname{dim} M / J M=1$.

Let $u_{1}, u_{2} \in A$ be a basis for $A$ over $B$, and write $u_{i}=b_{i} \oplus m_{i}$ for $i=1,2$. For some $c_{1}, c_{2} \in B$ we have $1=c_{1} u_{1}+c_{2} u_{2}$, so $1=c_{1} b_{1}+c_{2} b_{2}$ and $c_{1} m_{1}+c_{2} m_{2}=$ 0. Thus

$$
\left[\begin{array}{cc}
c_{1} & c_{2} \\
-b_{2} & b_{1}
\end{array}\right] \in M(2, B)
$$

is invertible, and if $m=-b_{2} m_{1}+b_{1} m_{2} \in M$, the computation

$$
\left[\begin{array}{cc}
c_{1} & c_{2} \\
-b_{2} & b_{1}
\end{array}\right]\left[\begin{array}{l}
u_{1} \\
u_{2}
\end{array}\right]=\left[\begin{array}{l}
1 \\
m
\end{array}\right]
$$

shows that $1, m$ also form a basis for $A$. This concludes the proof of (2).

We will show that $M$ is not a cyclic $B$-module; thus the theorem will be proved by (2). Let $\underline{A}=k[X, Y, Z] /\left(Z^{2}-X Y-1\right)$ and $V \subset A$ be the span of $x, y, z$. Let $\underline{B}=\left(V^{2}\right)$ and $\underline{M}=V+V^{3}+V^{5}+\ldots$, and let $A \stackrel{\frac{\pi}{\pi}}{\mapsto} \underline{A}$ be the algebra homomorphism determined by $x_{11}, x_{22} \mapsto z, x_{12} \mapsto x$, and $x_{21} \mapsto y$. It is clear that $\pi(B)=$ $\underline{B}$ and $\pi(M)=\underline{M}$. To prove that $M$ is not a cyclic $B$-module we need only to show that:

(3) $\underline{M}$ is not a cyclic $\underline{B}$-module.

First note that $\underline{A}$ is an extension of $k[X, Y]$, and is in fact a free $k[X, Y]$-module with basis $1, z$. Assume that $\underline{M}$ is cyclic with generator $\underline{m}$. Then $\underline{m}=p+q z$ where 
$p=p(X, Y), q=q(X, Y) \in k[X, Y]$, and $p$ is a linear combination of monomials of odd degree. In particular, $p \in k$ implies $p=0$. Now let $\underline{b}=p^{\prime}+q^{\prime} z \in \underline{B}$. Then $\underline{b} \underline{m}=\left(p p^{\prime}+q q^{\prime}(X Y+1)\right)+\left(p q^{\prime}+p^{\prime} q\right) z$, so since $X, z \in \underline{M}$, the equations

(a) $p p^{\prime}+q q^{\prime}(X Y+1)=X$,

(b) $p q^{\prime}+p^{\prime} q=0$,

(a') $p p^{\prime \prime}+q q^{\prime \prime}(X Y+1)=0$,

(b') $p q^{\prime \prime}+p^{\prime \prime} q=1$,

must have solutions for some $p^{\prime}, p^{\prime \prime}, q^{\prime}, q^{\prime \prime} \in k[X, Y]$. To show there are no solutions to the equations we may assume $k$ is algebraically closed.

First note that since $p \in k$ implies $p=0$, by $\left(\mathrm{b}^{\prime}\right)$ we deduce that $q \neq 0$. Let $\alpha \in k \backslash 0$ be such that $q(X, \alpha) \neq 0$. By virtue of $\left(\mathrm{a}^{\prime}\right)$ and $\left(\mathrm{b}^{\prime}\right)$ we have $p \mid q^{\prime \prime}(X Y+$ 1) and $q \mid p^{\prime \prime}$. By (a) $\alpha X+1$ does not divide $p(X, \alpha)$, so we conclude that $p(X, \alpha) \mid q^{\prime \prime}(X, \alpha)$. Write $p^{\prime \prime}(X, \alpha)=q(X, \alpha) u$ and $q^{\prime \prime}(X, \alpha)=p(X, \alpha) v$ where $u, v$ $\in k[X]$. Then by $\left(\mathrm{a}^{\prime}\right)$ we calculate $p(X, \alpha) q(X, \alpha)(u+v(\alpha X+1))=0$. Since $p(X, \alpha) \neq 0$ by (a) we have $u+v(\alpha X+1)=0$. Therefore by (b') we have $p(X, \alpha)^{2} v-q(X, \alpha)^{2} v(\alpha X+1)=1$, or

(4) $p(X, \alpha)^{2}-q(X, \alpha)^{2}(\alpha X+1) \in k$.

But this last equation is not possible since $p(x, \alpha)^{2}$ has even degree and $q(X, \alpha)^{2}(\alpha X+1)$ has odd degree. This proves (3), and the proof of the theorem is complete.

\section{REFERENCES}

1. Ulrich Oberst and Hans Jürgen Schneider, Untergruppen formeller gruppen von endlichen index, J. Algebra 31 (1974), 10-14.

2. D. E. Radford, Freeness (projectivity) criteria for Hopf algebras over Hopf subalgebras, J. Pure Appl. Algebra 11 (1977), 15-28.

3. __ Pointed Hopf algebras are free over Hopf subalgebras, J. Algebra 45 (1977), 266-273.

4. M. E. Sweedler, Hopf algebras, Benjamin, New York, 1969.

5. M. Takeuchi, A correspondence between Hopf ideals and subHopf algebras, Manuscripta Math. 7 (1972), 251-270.

6. Commutative Hopf algebras are projective over Hopf subalgebras, preprint.

7. preprint.

Department of Mathematics, University of Illinois, Chicago Crrcle, Illinois 60680

Current address: Department of Mathematics, Rutgers University, New Brunswick, New Jersey 08903 